\title{
SFRP5 acts as a mature adipocyte marker but not as a regulator in adipogenesis
}

\author{
Rui Wang ${ }^{1, *}$, Jie Hong, ${ }^{1, *}$, Ruixin Liu', ${ }^{1, *}$ Maopei Chen', Min Xu', Wiqiong Gu', Yifei Zhang', \\ Qinyun Ma', Feng Wang', Juan Shi', Jiqiu Wang', Weiqing Wang' and Guang Ning ${ }^{1,2}$ \\ ${ }^{1}$ Shanghai Clinical Center for Endocrine and Metabolic Diseases, Shanghai Institute of Endocrine and \\ Metabolic Diseases, Department of Endocrinology and Metabolism, Shanghai Key Laboratory for Endocrine \\ Tumors and E-Institutes of Shanghai Universities, Ruijin Hospital, Shanghai Jiao Tong University School of Medicine, \\ 197 Ruijin 2nd Road, Shanghai 200025, China \\ ${ }^{2}$ Laboratory for Endocrine and Metabolism, Institute of Health Sciences, Shanghai Institutes for Biological Sciences, \\ Chinese Academy of Sciences and Shanghai Jiao Tong University School of Medicine, Shanghai 200025, China \\ * $R$ Wang, J Hong and R Liu contributed equally to this work)
}

Correspondence should be addressed to G Ning or J Wang Emails

gning@sibs.ac.cn or jiqiuwang@163.com

\begin{abstract}
WNT/ $\beta$-catenin signalling is involved in regulating adipogenesis, and its dysregulation occurs in obesity. Secreted frizzled-related protein 5 (SFRP5) is a WNT protein inhibitor; however, its role in adipogenesis and obesity is controversial. In this study, we observed that SFRP5 mRNA levels were increased in the fat tissues of obese humans and mice. Sfrp 5 expression was gradually induced during differentiation of white and brown adipocytes and was highly increased in mature adipocytes rather than preadipocytes. However, the effects of the exogenous overexpression of Sfrp5 indicated that Sfrp5 may not directly regulate adipogenesis in vitro under the conditions studied. Moreover, SFRP5 did not inhibit the canonical WNT/ $\beta$-catenin signalling pathway in preadipocytes. Subsequently, we measured the levels of circulating SFRP5 in obese patients and non-obese subjects using ELISA and did not find any significant difference. Collectively, these findings indicate that Sfrp 5 represents a candidate for a mature adipocyte marker gene. Our data provide new evidence concerning the role of SFRP5 in adipogenesis of white and brown adipocytes and obesity.
\end{abstract}
Key Words
- adipogenesis
- adipocyte differentiation
- WNT signalling pathway
- $\beta$-catenin
- obesity

Journal of Molecular

Endocrinology

(2014) 53, 405-415

\section{Introduction}

The obesity epidemic presents one of the largest worldwide threats to overall health. The elucidation of the molecular mechanisms that are involved in adipogenesis is critical to the development of effective strategies for the prevention and treatment of obesity and its metabolic complications (Kelly et al. 2008, Flegal et al. 2012). Obesity is frequently associated with insulin resistance (IR), which in turn is linked to the development of many chronic diseases, such as type 2 diabetes (T2DM), hyperlipidaemia, hypertension and atherosclerosis (WHO Expert Consultation 2004). Adipogenesis is related to glucose and lipid metabolism and plays a role in IR (Rosen \& MacDougald 2006, Rosen \& Spiegelman 2006). Adipogenesis involves the sequential activation of a cascade of transcription factors that coordinate the expression of genes responsible for the adipogenic phenotype (Wu et al. 1999, Rosen et al. 2000). Results from gene functional studies indicate that evoked WNT/ $\beta$-catenin signalling in white and brown

Published by Bioscientifica Ltd 
preadipocytes leads to the maintenance of the undifferentiated state and prevents the induction of CCAAT/ enhancer-binding protein $\alpha$ (Cebpa) and peroxisome proliferator-activated receptor $\gamma$ (Pparg) (Ross et al. 2000, Kang etal. 2005, Rosen \& MacDougald 2006). In contrast, the disruption of WNT/ $\beta$-catenin signalling promotes adipogenesis (Bennett et al. 2002, Prestwich \& Macdougald 2007).

WNT10b, which has been the most extensively studied endogenous WNT in the regulation of adipocyte differentiation, decreases during adipogenesis preceding the downregulation of cytoplasmic $\beta$-catenin. Furthermore, the ectopic expression of WNT10b activates WNT/ $\beta$-catenin signalling and exhibits an anti-adipogenic action (Longo et al. 2004, Christodoulides et al. 2006, Aslanidi et al. 2007). Conversely, the extracellular antagonists of WNTs, such as dickkopfs, WNT inhibitory factor 1 and secreted frizzled-related proteins (SFRPs), have been shown to exert proadipogenic effects (Kawano \& Kypta 2003). SFRPs prevent downstream WNT signalling by binding to and sequestering WNT ligands in the extracellular space (Bovolenta et al. 2008). Among SFRPs, SFRP5 may interact with both canonical and non-canonical WNT signalling pathways (Li et al. 2008, Satoh et al. 2008) and might be an import metabolic regulator (Ouchi et al. 2010, Mori et al. 2012, Carstensen et al. 2014). Results from an in vitro study indicated that SFRP5 impairs insulin signalling under normal conditions, but reduces tumour necrosis factor $\alpha$-induced inflammation in primary human adipocytes (Carstensen et al. 2014). In murine mature adipocytes, SFRP5 expression is downregulated in druginduced IR and upregulated by insulin sensitisers (Lv et al. 2012). However, in vivo studies regarding the role of SFRP5 in adiposity have yielded conflicting data. Four groups reported increased Sfrp5 expression in genetic and dietinduced obese mouse models (Koza et al. 2006, Lagathu et al. 2009, Okada et al. 2009, Mori et al. 2012), whereas another group detected decreased Sfrp5 expression in $L e p^{o b / o b}$ and high-fat/high-sucrose diet-induced obese mice (Ouchi et al. 2010). As to the downstream signalling affected by SFRP5, Ouchi et al. (2010) reported that Sfrp5 deficiency in mice elevated phosphorylation of c-Jun N-terminal kinase, a downstream target of the non-canonical WNT signalling; however, the work from the MacDougald Laboratory indicated that SFRP5 inhibits canonical WNT signalling to suppress oxidative metabolism and stimulate adipogenesis (Mori et al. 2012). In contrast to the findings from studies using murine models, implicating SFRP5 in metabolic dysfunction, Ehrlund et al. (2013) indicated that SFRP5 in the WAT of Caucasians is not influenced by obesity. In light of the profound but conflicting effects of SFRP5 on adipogenesis and its unknown effect on brown adipocyte differentiation, we aimed to further investigate its function in adipogenesis.

SFRP5 is preferentially expressed in WAT and confined to adipocytes (Koza et al. 2006, Lagathu et al. 2009, Ouchi et al. 2010); however, whether it can be secreted into the blood for regulating metabolism is still a subject of debate. Ouchi et al. (2010) observed that detection of serum SFRP5 is only possible in mice after adenovirusmediated i.v. injection of Sfrp5. Similarly, Mori et al. (2012) demonstrated that SFRP5 is not easily detectable in whole-cell lysates or extracellular matrix (ECM) fractions except when overexpressed in HEK293T and 3T3L1 cells. Results from several studies have indicated that circulating SFRP5 is detectable in humans (Schulte et al. 2012, Carstensen et al. 2013, Hu et al. 2013a,b, Lu et al. 2013). However, both positive (Carstensen et al. 2013) and negative ( $\mathrm{Hu}$ et al. 2013a,b) associations between circulating SFRP5 concentrations and homeostasis model assessment of insulin resistance (HOMA-IR) were reported, whereas no statistically significant correlation between plasma SFRP5 levels and HOMA-IR was found in a Chinese cohort (Lu et al. 2013). Moreover, Ehrlund et al. (2013) reported that SFRP1, SFRP2 and SFRP4, but not SFRP3 or SFRP5, were actively secreted from human WAT and constituted adipokines. Additional investigations into the issue concerning whether SFRP5 acts in an endocrine manner are warranted.

In view of the inconclusive evidence regarding the role of SFRP5 in adiposity, we investigated the potential involvement of Sfrp 5 in adipogenesis and obesity. We tested the expression level of SFRP5 in human and murine adipose tissue and further explored its effects on differentiation of white and brown preadipocytes in vitro. Additionally, we determined whether SFRP5 exhibited extracellular localisation. Finally, we detected serum SFRP5 concentrations in obese and non-obese subjects using a replicable ELISA kit to test its application as a biomarker of obesity. Our study provides a better understanding of the influence of $S$ frp 5 on adipocyte differentiation.

\section{Materials and methods}

\section{Ethics statement}

This study was approved by the Institutional Review Board of the Ruijin Hospital, Shanghai Jiao Tong University School of Medicine, and was in accordance with the principle of the Helsinki Declaration II. Written informed consent was obtained from each participant.

Published by Bioscientifica Ltd. 


\section{Human adipose tissue samples}

Subcutaneous and visceral adipose tissues were obtained during surgical procedures from seven non-obese and 13 obese subjects. The exclusion criteria for subjects included the following: acute or chronic infectious or immunological diseases, cancer, hepatitis, hyper- or hypocortisolism, hyper- or hypothyroidism, hypertension, diabetes mellitus and a family history of the above diseases. The detailed characteristics of the subjects are presented in Supplementary Table 1, see section on supplementary data given at the end of this article.

\section{Animals}

Leptin-deficient $\left(L e p^{\mathrm{ob} / \mathrm{ob}}\right)$ mice have an excessive appetite and become profoundly obese. Lgr4 homozygous mutant $\left(L g r 4^{\mathrm{m} / \mathrm{m}}\right)$ mice display reduced adiposity and are resistant to high-fat diet-induced obesity (Wang etal. 2013). They served as obese and lean mouse models in this study. $L e p^{\mathrm{ob} / \mathrm{ob}}$ mice and their WT littermates were age- and sex-matched throughout the experiments. Male mice, 24 weeks of age, were used in the experiments ( $n=6-7 /$ group). $L g r 4^{\mathrm{m} / \mathrm{m}}$ mice were generated as described previously (Wang et al. 2012, 2013). $L g r 4^{\mathrm{m} / \mathrm{m}}$ mice show browning of white fat, which is more obvious when $\beta$-adrenergic signalling is activated by cold stress or isoprenaline treatment (Wang et al. 2013). To test gene expression in a lean mouse model regardless of experimental conditions, male $\operatorname{Lgr} 4^{\mathrm{m} / \mathrm{m}}$ mice, between 8 and 16 weeks of age, were subjected to cold room $\left(4^{\circ} \mathrm{C}\right)$ stress for 1 week or received injections of isoprenaline (7.5 mg/kg, Harvest Pharmaceutical, Shanghai, China) for 10 days before analysis as described previously (Wang et al. 2013). The dissected tissues were quickly frozen and then stored at $-80^{\circ} \mathrm{C}$. All of the animal experiments were approved by the Animal Care Committee of Shanghai Jiao Tong University School of Medicine.

\section{Cell culture}

3T3L1, C3H10T1/2 and HEK293T cell lines were obtained from the American Type Culture Collection (Manassas, VA, USA). The 3T3L1 preadipocyte line is the 'gold standard' for investigating differentiation of white preadipocytes (Tang \& Lane 2012). The pluripotent C3H10T1/2 cells serve as a faithful mesenchymal stem cell (MSC) model for genetic studies of the brown adipocyte developmental programme. The stromal vascular fraction of brown adipose tissue (BAT SVF) possesses a resident population of pluripotent MSCs, which enable it to serve as a model for the differentiation of brown adipocytes (Tang \& Lane 2012). The details of the isolation of SVF are presented in the Supplementary materials and methods, see section on supplementary data given at the end of this article. The HEK293T cell line is readily infected and is a widely used tool for studying protein function (Thomas \& Smart 2005). Murine 3T3L1 preadipocytes, C3H10T1/2 and HEK293T cells were grown in DMEM (Gibco) containing 10\% foetal bovine serum (FBS) (PAA, Pasching, Austria) and $100 \mathrm{U} / \mathrm{ml}$ penicillinstreptomycin (Gibco) at $37^{\circ} \mathrm{C}$ with $5 \% \mathrm{CO}_{2}$.

\section{Cell infection}

Adenoviral vectors overexpressing Sfrp5 (Ad-Sfrp5-Gfp) or empty vectors (Ad-Gfp) were manufactured to our specifications by Invitrogen Life Technologies. The cells were infected at $70 \%$ confluence.

\section{Differentiation of 3T3L1 and C3H10T1/2 cells into adipocytes}

Two days after confluence has been reached, the 3T3L1 preadipocytes were incubated with DMEM containing $0.5 \mathrm{mM}$ 3-isobutyl-1-methylxanthine (IBMX; Sigma), $5 \mu \mathrm{g} / \mathrm{ml}$ insulin (Eli Lilly) and $1 \mu \mathrm{M}$ dexamethasone (Sigma). After 2 days (day 2), the medium was replaced with DMEM containing only $10 \% \mathrm{FBS}$ and $5 \mu \mathrm{g} / \mathrm{ml}$ insulin. On day 4 , the medium was removed and a fresh medium with $10 \%$ FBS was added. The medium was changed every 2 days until the cells were collected. Differentiation of confluent C3H10T1/2 stem cells into brown adipocytes was performed in a growth medium supplemented with $0.5 \mathrm{mM}$ IBMX, $5 \mu \mathrm{g} / \mathrm{ml}$ insulin, $1 \mu \mathrm{M}$ dexamethasone, $50 \mathrm{nM}$ tri-iodothyronine (T3) (Sigma) and $5 \mu \mathrm{M}$ troglitazone (Sigma) for $48 \mathrm{~h}$. The cells were placed in a growth medium supplemented with insulin, T3 and troglitazone for another 6 days. The medium was changed every 2 days. mRNA was isolated on days $0,1,2,4,6$ and 8 .

\section{Oil Red $\mathrm{O}$ dye staining and triglyceride content}

Fully differentiated 3T3L1 adipocytes and SVF (8 days after induction) were collected and washed twice with PBS, then fixed in $4 \%$ formaldehyde for $20 \mathrm{~min}$ and washed twice with water. Oil Red O dye (Sigma) was dissolved in isopropanol and filtered. Cells were stained with the dye for $1 \mathrm{~h}$ and washed with water until the background became transparent. The images were recorded using a microscope (Olympus). 3T3L1 cell triglyceride levels were measured using a commercial kit (Kehua Bio-engineering Company, Shanghai, China) according to the manufacturer's instructions.

Published by Bioscientifica Ltd. 


\section{RNA isolation and real-time PCR}

Total RNA was extracted from the cells and adipose tissue samples using TRIzol reagent (Invitrogen) according to the manufacturer's instructions. The RNA integrity number (RIN) of samples was checked using an Agilent 2100 Bioanalyzer (Agilent Technologies, Santa Clara, CA, USA). Samples that had an RIN higher than 7.0 were eligible for the experiment and were adjusted to the same concentration. cDNA was generated from $1 \mu \mathrm{g}$ RNA using the RT System (Promega). The quantification of transcripts was carried out using the LC480 real-time PCR detection system (Roche Diagnostics, Penzberg, Germany) and SYBR Green I Supermix (Takara Bio Inc., Otsu, Shiga, Japan). The cycling conditions were as follows: $95^{\circ} \mathrm{C}$ for $30 \mathrm{~s}, 45$ cycles of $5 \mathrm{~s}$ at $95{ }^{\circ} \mathrm{C}$ followed by $30 \mathrm{~s}$ at $60^{\circ} \mathrm{C}$. The primers used in this study are presented in Supplementary Table 2, see section on supplementary data given at the end of this article.

\section{Protein preparation and western blotting}

3T3L1 preadipocytes were treated with 3 nM WNT3a (R\&D company, Minneapolis, MN, USA) and $1 \mu \mathrm{g} / \mathrm{ml}$ recombined murine SFRP5 protein (rmSFRP5) (R\&D company) individually or in combination. In another treatment, 3T3L1 preadipocytes were infected with Ad-Gfp or Ad-Sfrp5-Gfp respectively. After $72 \mathrm{~h}$, the nuclear and cytoplasmic protein extracts were isolated using NE-PER reagent according to the manufacturer's protocol (Pierce Biotechnology, Inc., Rockford, IL, USA). The protein concentration was determined by the BCA protein assay (Pierce Biotechnology, Inc.). Total protein was denatured by boiling, separated on a $10 \%$ SDS-PAGE gel and transferred onto a nitrocellulose membrane (Amersham Biosciences). After blocking in Odyssey blocking buffer (LI-COR, Lincoln, NE, USA) for $1 \mathrm{~h}$, the membranes were incubated overnight at $4{ }^{\circ} \mathrm{C}$ with primary antibodies directed against $\beta$-catenin (Cell Signaling Technology (CST), Danvers, MA, USA), $\alpha$-tubulin (CST), and Lamin B (Santa Cruz Biotechnology, Inc.). The secondary antibody used was anti-rabbit IgG (LI-COR) or anti-goat IgG (LI-COR), and the resulting bands were visualised using the Odyssey infrared imaging system (LI-COR).

\section{Localisation of SFRP5}

The separation of cell lysates, ECM, and conditioned medium fractions was performed as described previously (Lee et al. 2004, Mori et al. 2012). Briefly, the conditioned media were collected and concentrated by centrifuging at $3000 \boldsymbol{g}$ for $15 \mathrm{~min}$. The confluent HEK293T cells were released from the culture dishes by incubation with PBS containing $5 \mathrm{mMEDTA}$ at $37^{\circ} \mathrm{C}$ in an atmosphere of $5 \% \mathrm{CO}_{2}$ and were gently shaken every $10 \mathrm{~min}$, for a total of $40 \mathrm{~min}$. The cells were then verified to be completely released from the dishes using a microscope and collected. After washes, the remaining ECM components were extracted with $1 \times$ SDS Laemmli sample buffer containing 5\% $\beta$-mercaptoethanol and subjected to western bolt analysis.

\section{Measurement of SFRP5 in human serum}

A total of 160 young adults living in the eastern area of China (Han Chinese) were recruited for this study. A total of 80 patients with obesity were collected consecutively (from January 2010 to October 2012) from the specialised outpatient clinic for obesity in Ruijin Hospital, Shanghai Jiao Tong University School of Medicine. The inclusion criteria for patients with obesity were as follows: aged 18-30 years, with BMI $\geq 30 \mathrm{~kg} / \mathrm{m}^{2}$, previously untreated obesity, fasting blood glucose level lower than $7.0 \mathrm{mmol} / \mathrm{l}$ and $2 \mathrm{~h}$-postprandial glucose levels according to the oral glucose tolerance test (OGTT) lower than $11.1 \mathrm{mmol} / \mathrm{l}$. Patients with syndromatic obesity, diabetes mellitus, hyperand hypocortisolism, hyper- and hypothyroidism, growth hormone deficiency, severe hepatic or renal disease, acute or chronic infectious or immunological disease, cancer and pregnancy or breastfeeding, or being treated with glucoselowering drugs such as metformin were excluded from our study. Conforming to the age and sex distribution of the obese patients, 80 unrelated non-obese subjects were recruited from volunteers of Shanghai Jiao-Tong University School of Medicine. The inclusion criteria for controls were as follows: aged 18-30 years, with BMI $<23 \mathrm{~kg} / \mathrm{m}^{2}$ (WHO Expert Consultation 2004), currently physically well and with no past history of obesity. Non-obese subjects with clinical evidence of major diseases, hypertension, impaired glucose regulation and diabetes mellitus were excluded from our study. Blood samples were obtained after an overnight fast. Goat and mouse anti-human SFRP5 antibodies (Santa Cruz Biotechnology, Inc.) were used to produce the ELISA kit by Shanghai Senxiong Biotech (Shnghai, China). The assay was proven to be highly specific to human SFRP5 (intra-assay coefficient of variation $(\mathrm{CV})<10 \%$; inter-assay $\mathrm{CV}<15 \%$; analyticalsensitivity, $16 \mathrm{ng} / \mathrm{ml})$. The ELISA was performed according to the instructions from Shanghai Senxiong Biotech.

\section{Statistical analysis}

The Shapiro-Wilk test was used to test the normality of distribution for the data, and variables with $P$ values $<0.05$

Published by Bioscientifica Ltd. 
were logarithmically transformed for statistical analyses. The correlations between circulating SFRP5 levels and other metabolic parameters were calculated using Spearman's correlation coefficients. When we explored the correlation between SFRP5 and other metabolic parameters, we further adjusted for age and sex. All of the analyses were performed using SAS (version 9.3; SAS Institute, Inc., Cary, NC, USA). The data are presented as the means \pm s.E.m. values unless stated otherwise. Two-tailed Student's t-test or ANOVA followed by posthoc analysis were used for comparisons between groups, as appropriate. Differences were considered significant for $P$ values $<0.05$.

\section{Results}

\section{SFRP5 is increased in adipose tissues of obese humans and mice}

To investigate the role of SFRP5 in WAT biology, we first detected the mRNA expression levels of SFRP5 in visceral WAT (vWAT) and subcutaneous WAT (sWAT) from obese and non-obese subjects. The results of real-time PCR showed that the SFRP5 mRNA expression was robustly increased in vWAT from obese patients compared with non-obese controls (Fig. 1A). No significant difference in SFRP5 mRNA levels was detected in sWAT from obese patients compared with non-obese subjects (Fig. 1B).

We examined Sfrp5 mRNA levels in epididymal WAT (eWAT) and inguinal WAT (iWAT) of $L e p^{\mathrm{ob} / \mathrm{ob}}$ mice and WT mice. Sfrp5 displayed a higher level in eWAT of $L e p^{\mathrm{ob} / \mathrm{ob}}$ mice than in WT mice, although this trend did not reach statistical significance (Fig. 1C). Sfrp5 expression was significantly enhanced in iWAT of $L e p^{\mathrm{ob} / \mathrm{ob}}$ mice
(Fig. 1D). Consistent with the findings from studies using the obese mouse model, Sfrp 5 mRNA expression was reduced in eWAT of a lean mouse model, $L g r 4^{\mathrm{m} / \mathrm{m}}$ mice (Wang et al. 2013), under normal conditions, cold stress or isoprenaline treatment (Fig. 1E). Notably, isoprenaline treatment itself induced expression of $S f r p 5$ (Fig. 1E). These results indicate that SFRP5 is increased in the fat tissue of obese human vWAT and murine iWAT, which indicates that SFRP5 expression in WAT might be an indicator of obesity.

\section{Sfrp5 level is gradually elevated during differentiation of white and brown adipose tissue}

To study the biological function of $S f r p 5$ in adipogenesis, we assessed the changes in Sfrp5 expression during differentiation of white and brown adipocytes. We found that Sfrp5 expression incrementally increased during the differentiation of 3T3L1 cells, which was consistent with upregulation of the key transcription factors for adipogenesis, such as Cebpa and Pparg, and reached maximal expression 8 days after the initiation of differentiation (Fig. 2A, B and C).

During differentiation of C3H10T1/2 cells, although the groups were statistically underpowered, Sfrp5 mRNA demonstrated a tendency towards higher levels that paralleled the increased expression of brown-adipocytedifferentiation-associated genes, including PR domain containing 16 (Prdm16), Pparg coactivator 1 alpha (Ppargc1a) and cell death-inducing DNA fragmentation factor, alpha subunit-like effector A (Cidea) (Fig. 2D, E, F and $\mathrm{G})$. Moreover, we detected similar gene expression patterns during the differentiation of BAT SVF (Supplementary

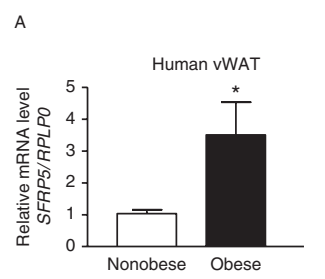

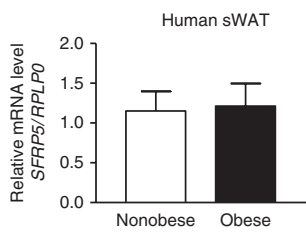

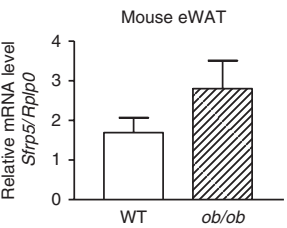

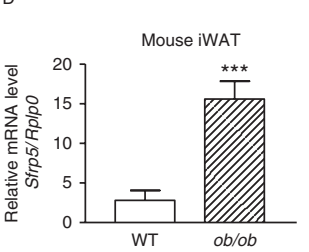

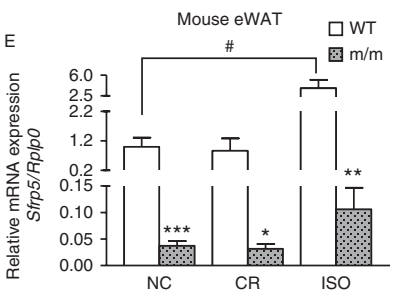

\section{Figure 1}

SFRP5 mRNA is increased with obesity in humans and mice. (A) and (B) SFRP5 mRNA was examined by real-time PCR in human visceral WAT (vWAT) and subcutaneous WAT (sWAT) from obese $(n=13)$ and non-obese $(n=7)$ subjects. ${ }^{*} P<0.05$ vs non-obese controls. Mean values are plotted with S.E.M. (C) and (D) Sfrp5 mRNA was examined by real-time PCR in epididymal WAT (eWAT) and inguinal adipose tissue (iWAT) of 24-week-old male wild-type (WT, $n=11)$ and $L e p^{o b / o b}(n=11)$ mice. WT, wild type; ob/ob, Lep $^{o b / o b}$. (E) Sfrp5 mRNA in eWAT of WT and $\operatorname{Lgr}^{\mathrm{m} / \mathrm{m}}(\mathrm{m} / \mathrm{m})$ mice under different conditions ( $n=6-9$ for wild type; and $n=5-8$ for $\mathrm{m} / \mathrm{m}$ ). NC, normal conditions; $\mathrm{CR}$, cold-room stimulation; ISO, isoprenaline treatment. WT, wild type; $\mathrm{m} / \mathrm{m}, \mathrm{Lgr} \mathrm{H}^{\mathrm{m} / \mathrm{m}}$. Differences among groups were examined by ANOVA followed by the Bonferroni's multiple comparison analysis. ${ }^{\#} P<0.05$ vs WT mice under normal conditions. For (C), (D) and (E), mean values are plotted with S.E.M.; ${ }^{*} P<0.05, * * P<0.01$ and $* * * P<0.001$ vs the corresponding WT mice. http://jme.endocrinology-journals.org DOI: 10.1530/JME-14-0037
(C) 2014 Society for Endocrinology Printed in Great Britain
Published by Bioscientifica Ltd 

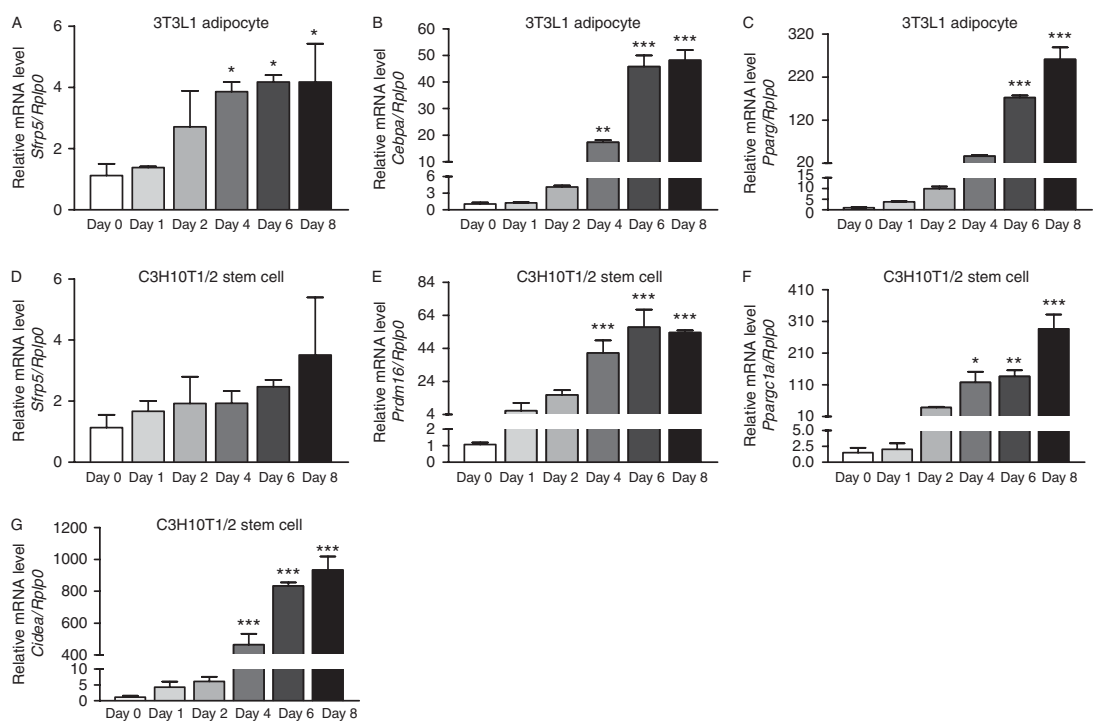

\section{Figure 2}

Sfrp5 mRNA is induced during differentiation of white and brown adipose tissue. Total RNA was isolated at the indicated time-points, subjected to real-time PCR and expression was normalised with respect to acidic ribosomal phosphoprotein PO (Rplp0) mRNA expression. Expression levels of (A) Sfrp5, (B) Cebpa and (C) Pparg were elevated incrementally during

Fig. 1A, B, C, D and E, see section on supplementary data given at the end of this article). Altogether, these results indicate that Sfrp5 is highly enriched in mature adipocytes rather than preadipocytes.

\section{Effects of Sfrp5 overexpression on adipocyte differentiation}

We then examined whether SFRP5 could promote adipogenesis of white or brown adipose tissue. 3T3L1 preadipocytes, C3H10T1/2 cells and BAT SVF were infected with adenoviral vectors encoding Sfrp5 (Ad-Sfrp5-Gfp) or empty vectors (Ad-Gfp). The total mRNA was isolated to determine the expression of adipocyte markers, and Oil Red O staining was performed to observe the morphology of lipid droplets in mature differentiated adipocytes. As shown in Fig. 3A and B, and Supplementary Fig. 1F, Sfrp5 was highly overexpressed in 3T3L1, C3H10T1/2 cells and BAT SVF after infection with Ad-Sfrp5-Gfp. Exogenous overexpression of $S f r p 5$ did not result in increased expression of white-adipocyte-specific marker genes, including resistin (Retn), homeobox C9 (Hoxc9), Transcription factor 21 (Tcf21), Inhibin beta B (Inhbb) and Serine or cysteine proteinase inhibitor, clade A, member 3K (Serpina3k), or brown adipocyte-specific marker genes, including Prdm16, Ppargc1a and Cidea as expected (Fig. 3A and B, differentiation of 3T3L1 adipocytes. Expression levels of (D) Sfrp5, (E) Prdm16, (F) Ppargc1a and (G) Cidea were elevated incrementally during differentiation of C3H10T1/2 cells. Mean values are plotted with S.E.M. $(n=3)$. Differences among groups were calculated by ANOVA followed by post-hoc Dunnett's test. ${ }^{*} P<0.05, * * P<0.01$ and ${ }^{* * *} P<0.001$ vs day 0 .

Supplementary Fig. 1F). Oil Red O staining revealed that Sfrp5 overexpression did not change the number of lipid droplets in differentiated 3T3L1 (Fig. 3C), C3H10T1/2 cells (data not shown) or BAT SVF (Supplementary Fig. 1G). Thus, although Sfrp5 is increased in fat tissues with obesity and during adipogenesis, it seems that SFRP5 does not affect adipogenesis of white or brown adipose tissue.

\section{SFRP5 does not affect WNT/ $\beta$-catenin signalling in preadipocytes}

SFRP5 has been identified as an inhibitor of WNT signalling; however, its role in canonical WNT signalling in preadipocytes remains undetermined (Ouchi et al. 2010, Mori et al. 2012). 3T3L1 preadipocytes were treated with rmSFRP5 and canonical WNT signalling ligand WNT3a, individually or in combination. As shown in Fig. 3D, WNT3a treatment resulted in an increased translocation of $\beta$-catenin to the nucleus, whereas rnSFRP5 treatment did not change basal or WNT3a-induced nuclear $\beta$-catenin levels. Consistent with the results of rmSFRP5 treatment, the Ad-Sfrp5-Gfp-infected 3T3L1 cells displayed an unaltered amount of $\beta$-catenin in either the cytoplasm or the nucleus compared with controls. Altogether, these findings indicate that SFRP5 does not appear to regulate $\mathrm{WNT} / \beta$-catenin signalling in preadipocytes. 

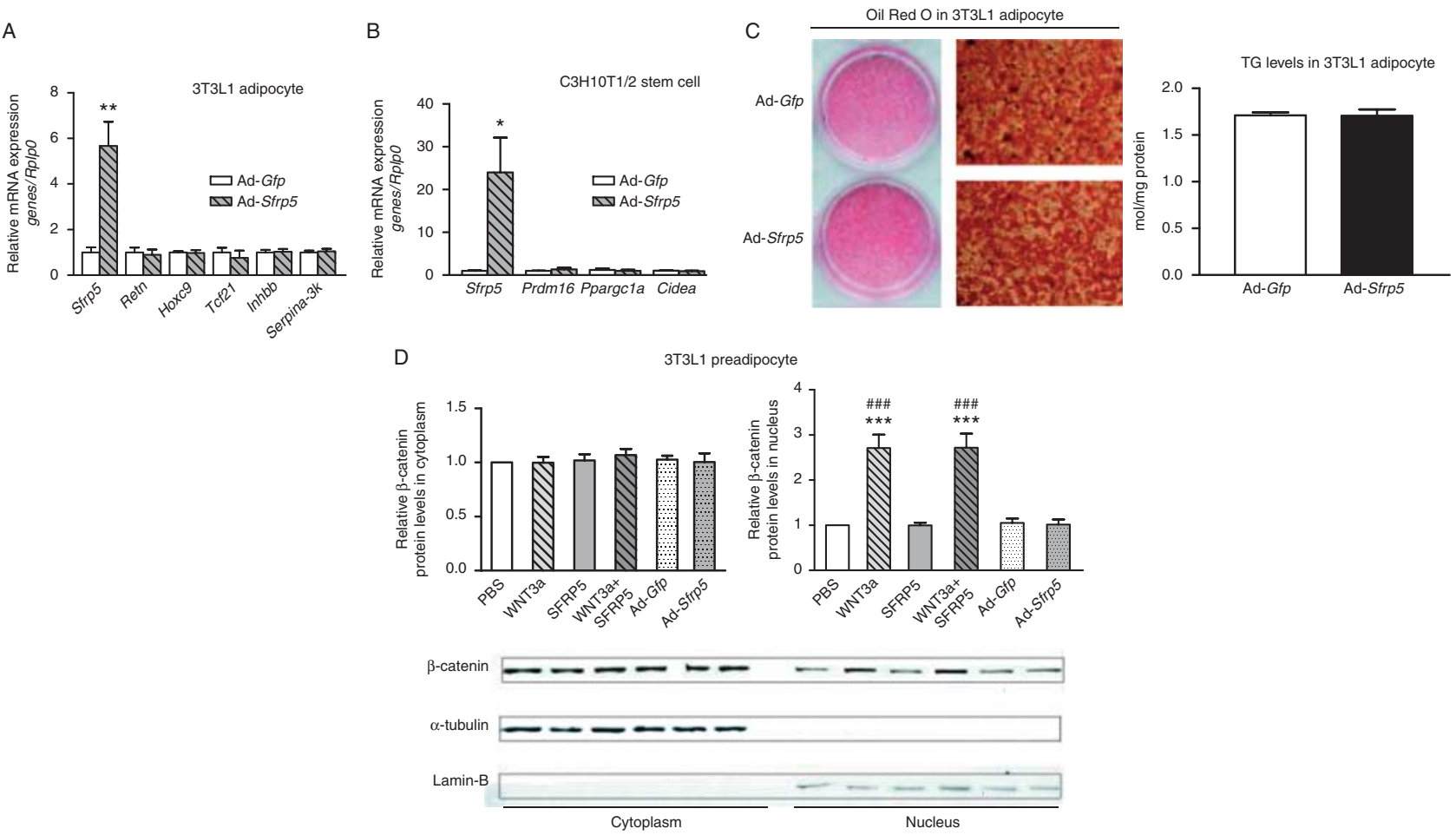

Figure 3

Sfrp5 overexpression does not affect 3T3L1 and C3H10T1/2 cell differentiation or the translocation of $\beta$-catenin to the nucleus in 3T3L1 preadipocytes. (A) Exogenous overexpression of Sfrp5 did not alter expressions of Retn, Hoxc9, Tcf21, Inhbb and Serpina3k in 3T3L1 adipocytes after infection with Sfrp5 (day 8 after differentiation; $n=5$ ). (B) Exogenous overexpression of Sfrp5 did not alter expressions of Prdm16, Ppargc1a and Cidea in C3H10T1/2 stem cells after infection with Sfrp5 (day 8 after differentiation; $n=3$ ). (C) Sfrp5 overexpression did not affect adipocyte differentiation. (Left panel) Pictures of Oil Red O (ORO) staining showing lipid accumulation in 3T3L1 adipocytes. Objective magnification, $\times 10$. (Right panel) Triglyceride (TG) content of 3T3L1 adipocytes (day 8 after differentiation; $n=5$ ). Ad-Gfp, 3T3L1 adipocytes infected with an empty adenoviral vector; Ad-Sfrp5, 3T3L1 adipocytes infected with an adenoviral

\section{Serum SFRP5 levels cannot be used to distinguish obese patients from non-obese subjects}

On the basis of our data, SFRP5 is highly enriched in mature adipocytes and adipose tissues of obese humans and mice. Thus, SFRP5 might be a circulating indicator of obese patients. To address this issue, we measured serum SFRP5 concentrations of 80 non-diabetic obese and 80 age- and sex-matched non-obese subjects (Supplementary Table 3, see section on supplementary data given at the end of this article). The specificity of the antibodies used in ELISA was tested by western blot experiments (Fig. 4A and $\mathrm{B})$. The standard curve confirmed a wide detection range between 0 and $800 \mathrm{ng} / \mathrm{ml}$ (Fig. 4C). SFRP5 was detectable in non-obese $(41.47 \pm 2.26 \mathrm{ng} / \mathrm{ml})$ and obese vector encoding Sfrp5. For (A), (B) and (C), mean values are plotted with s.E.M.; $* P<0.05$ and $* * P<0.01$ vs Ad-Gfp. (D) SFRP5 does not affect $\beta$-catenin translocation in 3T3L1 preadipocytes. (Upper panel) Quantification of $\beta$-catenin in cytoplasm and nucleus of 3T3L1 preadipocytes. (Lower panel) 3T3L1 preadipocytes were treated with PBS, WNT3a and recombined mouse SFRP5 individually or in combination. Ad-Gfp, 3T3L1 preadipocytes infected with an empty adenoviral vector; Ad-Sfrp5, 3T3L1 preadipocytes infected with an adenoviral vector encoding Sfrp5. Mean values are plotted with S.E.M. $(n=5)$. Differences among groups were calculated by ANOVA followed by the Bonferroni's multiple comparison analysis. ${ }^{* *} P<0.001$ vs preadipocytes treated with PBS; and ${ }^{\# \#} P<0.001$ vs preadipocytes treated with recombined mouse SFRP5.

subjects $(44.84 \pm 2.36 \mathrm{ng} / \mathrm{ml})$; however, there was no statistically significant difference between the two groups (Fig. 4D). Additionally, no significant associations were found between the circulating SFRP5 levels and anthropometric measurements, including BMI, waist circumference, percentage body fat, the biochemical characteristics and HOMA-IR (Supplementary Table 4, see section on supplementary data given at the end of this article).

\section{SFRP5 is incorporated into the ECM}

SFRP1 and SFRP2 are thought to act locally by remaining tightly associated with the ECM. To determine whether SFRP5 exhibits similar extracellular localisation, HEK293T cells were infected with Ad-Gfp or Ad-Sfrp5-Gfp. Cell lysates,

Published by Bioscientifica Ltd. 

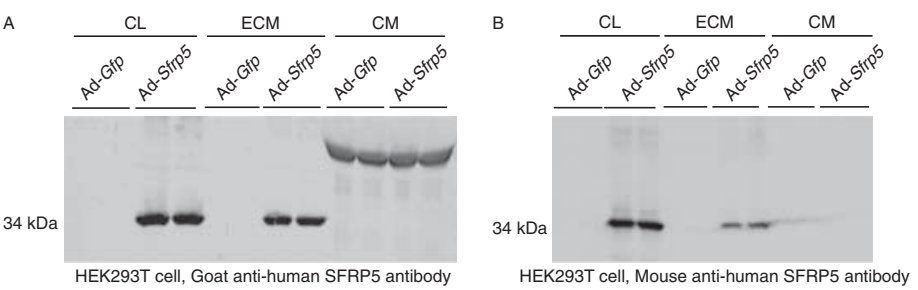
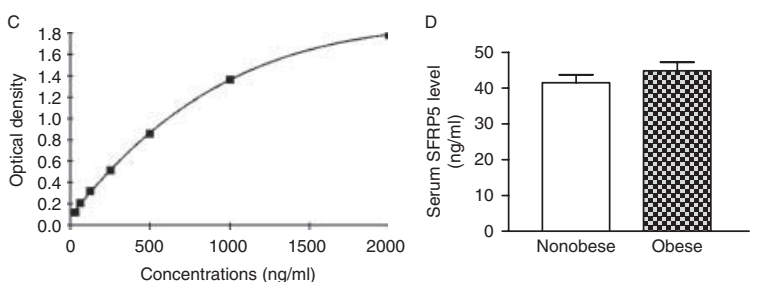

Figure 4

SFRP5 is tightly associated with the extracellular matrix (ECM), and serum SFRP5 concentrations cannot be used to distinguish obese patients from non-obese subjects. ( $A$ and B) Expression and localisation of SFRP5 in vitro. SFRP5 protein is secreted but remains associated with the ECM. Western blot confirming the specificity of goat $(A)$ and mouse $(B)$ anti-human SFRP5 antibodies used in ELISA. Ad-Gfp, HEK293T cells infected with an empty

ECM and conditioned medium fractions were collected and subjected to immunoblot analysis. When overexpressed, the SFRP5 protein was detected in the cell lysates and ECM fractions but not in the conditioned media (Fig. $4 \mathrm{~A}$ and $\mathrm{B}$ ). These results indicate that, upon secretion from the cells, SFRP5 is tightly associated with the ECM and probably functions primarily in an autocrine or paracrine manner.

\section{Discussion}

In the last decade, emerging evidence has indicated an important role for the WNT signalling pathway in adipogenesis (Ross et al. 2000, Kennell \& MacDougald 2005). Research regarding the regulation of Sfrp5 and obesity demonstrates conflicting results. A signal-net array examined in our previous study indicated a notable decline in Sfrp5 mRNA expression in lean $L g r 4^{\mathrm{m} / \mathrm{m}}$ mice compared with their normal-weight littermates (J Wang, R Liu, F Wang, M Chen, $\mathrm{J}$ Hong and G Ning, unpublished observations). Consistently, results obtained from compelling experiments indicate that $S f r p 5$ is highly induced in mouse models of genetic and diet-induced obesity, such as $L e p^{\mathrm{ob} / \mathrm{ob}}$, leptin receptordeficient $\left(L e p r^{\mathrm{db} / \mathrm{db}}\right)$ and C57BL/6J mice exposed to a high-fat diet (Koza et al. 2006, Lagathu et al. 2009, Okada et al. 2009, Mori et al. 2012), and support a model of adipogenesis in which SFRP5 inhibits $\mathrm{WNT} / \beta$-catenin signalling to stimulate adipocyte growth (Lagathu et al. 2009, Lv et al. 2012, Mori et al. 2012). In contrast, Ouchi et al. (2010) reported downregulation of SFRP5 under these conditions and proposed that SFRP5 neutralised non-canonical JNK activation by WNT5a in murine adipocytes. In this study, we observed that exogenous SFRP5 treatment did not change basal or WNT3a-induced nuclear $\beta$-catenin levels, which indicates that SFRP5 does not work through canonical $\mathrm{WNT} / \beta$-catenin signalling in preadipocytes and adenoviral vector; Ad-Sfrp5, HEK293T cells infected with an adenoviral vector encoding Sfrp5; CL, cell lysates; $\mathrm{CM}$, conditioned media. (C) The standard curve confirmed a wide detection range of the ELISA Kit. (D) Serum samples were obtained after an overnight fast and SFRP5 levels were measured using ELISA. SFRP5 concentrations are similar in obese $(n=80)$ and non-obese $(n=80)$ subjects. Mean values are plotted with S.E.M.

that other signalling, such as WNT5a, might be involved (Kawano \& Kypta 2003, Ouchi et al. 2010, Cruciat \& Niehrs 2013). Further mechanism studies should also investigate the non-classical roles of SFRPs, including the interaction of SFRP5 with the cell-surface receptors and many other signalling networks, such as transforming growth factor beta, BMPs and FGFs (Lee et al. 2006, Placencio et al. 2008, Cruciat \& Niehrs 2013).

In this study, we clarified that SFRP5 was increased overall in fat tissues under an obese state, which indicates that SFRP5 may play an important role in the development of obesity. Nevertheless, our results indicate a different expression pattern of SFRP5 between humans and mice. This might be due, in part, to the different features of fat depots between humans and mice. For example, a recent publication reports that the functional profile of human sWAT is similar to that of mouse mesenteric and perigonadal depots (Cypess et al. 2013).

To date, studies of the action of Sfrp 5 in differentiation of brown adipocytes are limited. Our present data first revealed that Sfrp5 mRNA displayed a tendency towards higher levels during differentiation of brown adipocytes in vitro, paralleling the increased expression of brown adipocyte differentiation-associated genes. Consistent with our results, previous in vitro evidence has indicated a gradual induction of SFRP5 expression during differentiation of white adipose tissue (Lv et al. 2012, Mori et al. 2012). However, we found that the exogenous expression of Sfrp5 leads to unaltered expression levels of Cebpa, Pparg (data not shown) and white- or brown-adipocyte-specific marker genes. The MacDougald Lab also failed to verify a regulatory role for Sfrp5 in ear MSC differentiation, with no marked influence of Sfrp5 ablation in the morphology of lipid droplets or expression of common adipocyte marker genes such as Pparg and Fabp4 observed (Mori et al. 2012). It seems that these results cannot explain the decreased fat weight in the

Published by Bioscientifica Ltd. 
Sfrp5-deficient mouse model (Mori et al. 2012), which might be caused by the fact that, although in vitro adipogenesis can be synchronously induced by exposure to a defined adipogenic cocktail, in vivo adipose tissue expansion is regulated by a combination of local and endocrine factors.

Our previous results have indicated that $L g r 4^{\mathrm{m} / \mathrm{m}}$ mice displayed reduced body weight when compared with WT mice. $L g r 4^{\mathrm{m} / \mathrm{m}}$ mice showed browning of white fat, which was more obvious when being activated by cold stress or isoprenaline treatment (Wang et al. 2013). In this study, we detected SFRP5 expression in WT and $L g r 4^{\mathrm{m} / \mathrm{m}}$ mice under different conditions to demonstrate a reduction in SFRP5 in a lean mouse model regardless of experimental conditions. Notably, we observed that isoprenaline treatment but not cold-room stimulation induced expression of Sfrp5. To our knowledge, both cold-room stimulation and isoprenaline treatment activate $\beta$-adrenergic signalling. The detailed molecular mechanism as to how isoprenaline regulates SFRP5 expression is not clear and future studies are needed to address this issue.

WAT appeared to be one of the major sources of SFRP5 in obese mice (Ouchi et al. 2010, Mori et al. 2012). It is important to establish whether SFRP5 influences metabolism in distal tissues and organs through an endocrine mechanism, similar to leptin or adiponectin. In line with observations in adipocytes (Mori et al. 2012), we found that, when overexpressed, SFRP5 was incorporated into the ECM, and its effects may be more relevant locally. As SFRP5 has been reported to be highly expressed in retinal pigment epithelium and be moderately expressed in the pancreas (Chang et al. 1999), the circulating SFRP5 concentrations might not reflect their expression levels in WAT. This notion is corroborated by our findings that the serum SFRP5 levels were clearly measurable but could not be used to distinguish obese patients from non-obese subjects, which is consistent with the findings of two other studies on Caucasians (Schulte et al. 2012, Carstensen et al. 2013).

In contrast with ours and others' data (Schulte et al. 2012, Hu et al. 2013a,b), Carstensen et al. (2013) have reported a much wider range of circulating SFRP5 concentrations in mostly overweight and obese Caucasians. It is likely that this disparity is partly related to the different clinical characteristics of these studies, including glycaemia levels, blood pressure, certain medications, other diseases and the variance in the definition of obesity. Although both positive (Carstensen et al. 2013) and negative (Hu et al. 2013a,b) associations between circulating SFRP5 concentrations and HOMA-IR have been reported, we did not observe a statistically significant correlation between serum SFRP5 levels and HOMA-IR in our cohort consisting of a total of 160 young adults with a broad range of BMI. The reasons for this discrepancy might be a comparatively healthier glucose metabolic state (only 21 obese patients had impaired glucose regulation), a much younger age and a higher degree of obesity in our study population. This study focused on obesity in young adults, so that our results may not be fully generalisable to older populations. Moreover, another study on Chinese subjects including 82 subjects with T2DM and 42 controls also detected no statistically significant correlation between plasma SFRP5 concentrations and HOMA-IR $(r=0.148, P=0.101)$. Unfortunately, this study did not mention whether this association was different between non-obese and obese subjects (Lu et al. 2013).

In summary, our findings demonstrate that the change in circulating SFRP5 levels may differ from the elevated Sfrp5 expression in fat tissue in an obese state. Moreover, Sfrp5 is enriched in both white and brown mature adipocytes, but the exogenous overexpression of Sfrp5 does not affect adipogenesis, which indicated that, as an adipokine, SFRP5 gene upregulation appears to be caused by obesity and that it might thus be a novel mature adipocyte marker gene.

\section{Supplementary data}

This is linked to the online version of the paper at http://dx.doi.org/10.1530/ JME-14-0037.

\section{Declaration of interest}

The authors declare that there is no conflict of interest that could be perceived as prejudicing the impartiality of the research reported.

\section{Funding}

This work was supported in part by grants from the National Natural Science Foundation of China (No. 81270931, 81270877 and 81100601).

\section{Acknowledgements}

The authors thank Yingchao Chen, Lin Miao, Minglan Yang and Yingkai Sun for the collection of serum from subjects.

\section{References}

Aslanidi G, Kroutov V, Philipsberg G, Lamb K, Campbell-Thompson M, Walter GA, Kurenov S, Ignacio Aguirre J, Keller P, Hankenson K et al. 2007 Ectopic expression of Wnt10b decreases adiposity and improves glucose homeostasis in obese rats. American Journal of Physiology. Endocrinology and Metabolism 293 E726-E736. (doi:10.1152/ajpendo. 00248.2007)

Published by Bioscientifica Ltd. 
Bennett CN, Ross SE, Longo KA, Bajnok L, Hemati N, Johnson KW, Harrison SD \& MacDougald OA 2002 Regulation of Wnt signaling during adipogenesis. Journal of Biological Chemistry 277 30998-31004. (doi:10.1074/jbc.M204527200)

Bovolenta P, Esteve P, Ruiz JM, Cisneros E \& Lopez-Rios J 2008 Beyond Wnt inhibition: new functions of secreted frizzled-related proteins in development and disease. Journal of Cell Science 121 737-746. (doi:10.1242/jcs.026096)

Carstensen M, Herder C, Kempf K, Erlund I, Martin S, Koenig W, Sundvall J, Bidel S, Kuha S, Roden M et al. 2013 Sfrp5 correlates with insulin resistance and oxidative stress. European Journal of Clinical Investigation 43 350-357. (doi:10.1111/eci.12052)

Carstensen M, Wiza C, Rohrig K, Fahlbusch P, Roden M, Herder C \& Ouwens DM 2014 Effect of Sfrp5 on cytokine release and insulin action in primary human adipocytes and skeletal muscle cells. PLOS ONE 9 e85906. (doi:10.1371/journal.pone.0085906)

Chang JT, Esumi N, Moore K, Li Y, Zhang S, Chew C, Goodman B, Rattner A, Moody S, Stetten G et al. 1999 Cloning and characterization of a secreted frizzled-related protein that is expressed by the retinal pigment epithelium. Human Molecular Genetics 8 575-583. (doi:10.1093/hmg/8.4.575)

Christodoulides C, Scarda A, Granzotto M, Milan G, Dalla Nora E, Keogh J, De Pergola G, Stirling H, Pannacciulli N, Sethi JK et al. 2006 WNT10B mutations in human obesity. Diabetologia 49 678-684. (doi:10.1007/ s00125-006-0144-4)

Cruciat CM \& Niehrs C 2013 Secreted and transmembrane Wnt inhibitors and activators. Cold Spring Harbor Perspectives in Biology 5 a015081. (doi:10.1101/cshperspect.a015081)

Cypess AM, White AP, Vernochet C, Schulz TJ, Xue R, Sass CA, Huang TL, Roberts-Toler C, Weiner LS, Sze C et al. 2013 Anatomical localization, gene expression profiling and functional characterization of adult human neck brown fat. Nature Medicine 19 635-639. (doi:10.1038/nm. 3112) (doi:10.1038/nm.3112)

Ehrlund A, Mejhert N, Lorente-Cebrian S, Astrom G, Dahlman I, Laurencikiene J \& Ryden M 2013 Characterization of the Wnt inhibitors secreted frizzled-related proteins (SFRPs) in human adipose tissue. Journal of Clinical Endocrinology and Metabolism 98 E503-E508. (doi:10.1210/jc.2012-3416)

Flegal KM, Carroll MD, Kit BK \& Ogden CL 2012 Prevalence of obesity and trends in the distribution of body mass index among US adults, 1999-2010. Journal of the American Medical Association 307 491-497. (doi:10.1001/jama.2012.39)

Hu W, Li L, Yang M, Luo X, Ran W, Liu D, Xiong Z, Liu H \& Yang G 2013 a Circulating Sfrp5 is a signature of obesity-related metabolic disorders and is regulated by glucose and liraglutide in humans. Journal of Clinical Endocrinology and Metabolism 98 290-298. (doi:10.1210/jc.2012-2466)

Hu Z, Deng H \& Qu H 2013b Plasma SFRP5 levels are decreased in Chinese subjects with obesity and type 2 diabetes and negatively correlated with parameters of insulin resistance. Diabetes Research and Clinical Practice 99 391-395. (doi:10.1016/j.diabres.2012.11.026)

Kang S, Bajnok L, Longo KA, Petersen RK, Hansen JB, Kristiansen K \& MacDougald OA 2005 Effects of Wnt signaling on brown adipocyte differentiation and metabolism mediated by PGC-1 $\alpha$. Molecular and Cellular Biology 25 1272-1282. (doi:10.1128/MCB.25.4.1272-1282.2005)

Kawano Y \& Kypta R 2003 Secreted antagonists of the Wnt signalling pathway. Journal of Cell Science 116 2627-2634. (doi:10.1242/jcs.00623)

Kelly T, Yang W, Chen CS, Reynolds K \& He J 2008 Global burden of obesity in 2005 and projections to 2030. International Journal of Obesity 32 1431-1437. (doi:10.1038/ijo.2008.102)

Kennell JA \& MacDougald OA 2005 Wnt signaling inhibits adipogenesis through $\beta$-catenin-dependent and -independent mechanisms. Journal of Biological Chemistry 280 24004-24010. (doi:10.1074/ jbc.M501080200)

Koza RA, Nikonova L, Hogan J, Rim JS, Mendoza T, Faulk C, Skaf J \& Kozak LP 2006 Changes in gene expression foreshadow diet-induced obesity in genetically identical mice. PLoS Genetics 2 e81. (doi:10.1371/journal.pgen.0020081)
Lagathu C, Christodoulides C, Virtue S, Cawthorn WP, Franzin C, Kimber WA, Nora ED, Campbell M, Medina-Gomez G, Cheyette BN et al. 2009 Dact1, a nutritionally regulated preadipocyte gene, controls adipogenesis by coordinating the Wnt/ $\beta$-catenin signaling network. Diabetes 58 609-619. (doi:10.2337/db08-1180)

Lee JL, Lin CT, Chueh LL \& Chang CJ 2004 Autocrine/paracrine secreted frizzled-related protein 2 induces cellular resistance to apoptosis: a possible mechanism of mammary tumorigenesis. Journal of Biological Chemistry 279 14602-14609. (doi:10.1074/jbc.M309008200)

Lee HX, Ambrosio AL, Reversade B \& De Robertis EM 2006 Embryonic dorsal-ventral signaling: secreted frizzled-related proteins as inhibitors of tolloid proteinases. Cell 124 147-159. (doi:10.1016/j.cell.2005.12.018)

Li Y, Rankin SA, Sinner D, Kenny AP, Krieg PA \& Zorn AM 2008 Sfrp5 coordinates foregut specification and morphogenesis by antagonizing both canonical and noncanonical Wnt11 signaling. Genes \& Development 22 3050-3063. (doi:10.1101/gad.1687308)

Longo KA, Wright WS, Kang S, Gerin I, Chiang SH, Lucas PC, Opp MR \& MacDougald OA 2004 Wnt10b inhibits development of white and brown adipose tissues. Journal of Biological Chemistry 279 35503-35509. (doi:10.1074/jbc.M402937200)

Lu YC, Wang CP, Hsu CC, Chiu CA, Yu TH, Hung WC, Lu LF, Chung FM, Tsai IT, Lin HC et al. 2013 Circulating secreted frizzled-related protein 5 (Sfrp5) and wingless-type MMTV integration site family member 5a (Wnt5a) levels in patients with type 2 diabetes mellitus. Diabetes/ Metabolism Research and Reviews 29 551-556. (doi:10.1002/dmrr.2426)

Lv C, Jiang Y, Wang H \& Chen B 2012 Sfrp5 expression and secretion in adipocytes are up-regulated during differentiation and are negatively correlated with insulin resistance. Cell Biology International 36 851-855. (doi:10.1042/CBI20120054)

Mori H, Prestwich TC, Reid MA, Longo KA, Gerin I, Cawthorn WP, Susulic VS, Krishnan V, Greenfield A \& Macdougald OA 2012 Secreted frizzled-related protein 5 suppresses adipocyte mitochondrial metabolism through WNT inhibition. Journal of Clinical Investigation 122 2405-2416. (doi:10.1172/JCI63604)

Okada Y, Sakaue H, Nagare T \& Kasuga M 2009 Diet-induced up-regulation of gene expression in adipocytes without changes in DNA methylation. Kobe Journal of Medical Sciences 54 E241-E249.

Ouchi N, Higuchi A, Ohashi K, Oshima Y, Gokce N, Shibata R, Akasaki Y, Shimono A \& Walsh K 2010 Sfrp5 is an anti-inflammatory adipokine that modulates metabolic dysfunction in obesity. Science 329 454-457. (doi:10.1126/science.1188280)

Placencio VR, Sharif-Afshar AR, Li X, Huang H, Uwamariya C, Neilson EG, Shen MM, Matusik RJ, Hayward SW \& Bhowmick NA 2008 Stromal transforming growth factor- $\beta$ signaling mediates prostatic response to androgen ablation by paracrine Wnt activity. Cancer Research 68 4709-4718. (doi:10.1158/0008-5472.CAN-07-6289)

Prestwich TC \& Macdougald OA $2007 \mathrm{Wnt} / \beta$-catenin signaling in adipogenesis and metabolism. Current Opinion in Cell Biology 19 612-617. (doi:10.1016/j.ceb.2007.09.014)

Rosen ED \& MacDougald OA 2006 Adipocyte differentiation from the inside out. Nature Reviews. Molecular Cell Biology 7 885-896. (doi:10.1038/nrm2066)

Rosen ED \& Spiegelman BM 2006 Adipocytes as regulators of energy balance and glucose homeostasis. Nature 444 847-853. (doi:10.1038/ nature05483)

Rosen ED, Walkey CJ, Puigserver P \& Spiegelman BM 2000 Transcriptional regulation of adipogenesis. Genes \& Development 14 1293-1307. (doi:10.1101/gad.14.11.1293)

Ross SE, Hemati N, Longo KA, Bennett CN, Lucas PC, Erickson RL \& MacDougald OA 2000 Inhibition of adipogenesis by Wnt signaling. Science 289 950-953. (doi:10.1126/science.289.5481.950)

Satoh W, Matsuyama M, Takemura H, Aizawa S \& Shimono A 2008 Sfrp1, Sfrp2, and Sfrp5 regulate the Wnt/ $\beta$-catenin and the planar cell polarity pathways during early trunk formation in mouse. Genesis 46 92-103. (doi:10.1002/dvg.20369) 
Schulte DM, Muller N, Neumann K, Oberhauser F, Faust M, Gudelhofer H, Brandt B, Krone W \& Laudes M 2012 Pro-inflammatory wnt5a and antiinflammatory sFRP5 are differentially regulated by nutritional factors in obese human subjects. PLoS ONE 7 e32437. (doi:10.1371/journal. pone.0032437)

Tang QQ \& Lane MD 2012 Adipogenesis: from stem cell to adipocyte. Annual Review of Biochemistry 81 715-736. (doi:10.1146/annurevbiochem-052110-115718)

Thomas P \& Smart TG 2005 HEK293 cell line: a vehicle for the expression of recombinant proteins. Journal of Pharmacological and Toxicological Methods 51 187-200. (doi:10.1016/j.vascn.2004.08.014)

Wang J, Li X, Ke Y, Lu Y, Wang F, Fan N, Sun H, Zhang H, Liu R, Yang J et al. 2012 GPR48 increases mineralocorticoid receptor gene expression. Journal of the American Society of Nephrology 23 281-293. (doi:10.1681/ASN.2011040351)

Wang J, Liu R, Wang F, Hong J, Li X, Chen M, Ke Y, Zhang X, Ma Q, Wang R et al. 2013 Ablation of LGR4 promotes energy expenditure by driving white-to-brown fat switch. Nature Cell Biology 15 1455-1463. (doi:10.1038/ncb2867)

WHO Expert Consultation 2004 Appropriate body-mass index for Asian populations and its implications for policy and intervention strategies. Lancet 363 157-163. (doi:10.1016/S0140-6736(03)15268-3)

Wu Z, Rosen ED, Brun R, Hauser S, Adelmant G, Troy AE, McKeon C, Darlington GJ \& Spiegelman BM 1999 Cross-regulation of C/EBP $\alpha$ and PPAR $\gamma$ controls the transcriptional pathway of adipogenesis and insulin sensitivity. Molecular Cell 3 151-158. (doi:10.1016/S1097-2765(00)80306-8)

Received in final form 25 September 2014

Accepted 8 October 2014

Accepted Preprint published online 16 October 2014
Published by Bioscientifica Ltd. 